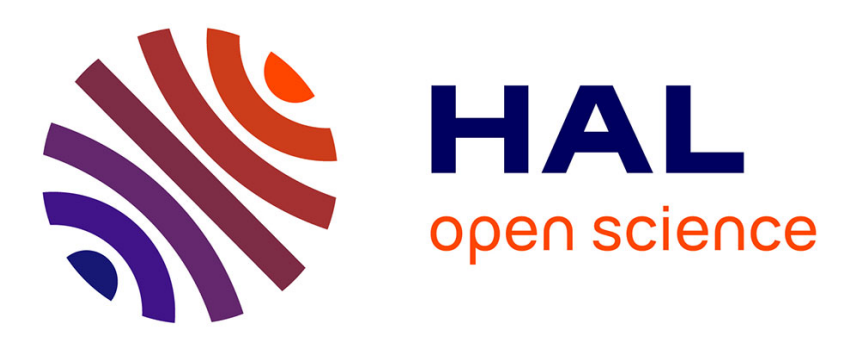

\title{
Macrostructural abnormalities in Korsakoff syndrome compared with uncomplicated alcoholism.
}

Anne-Lise Pitel, Gaël Chételat, Anne-Pascale Le Berre, Béatrice Desgranges, Francis Eustache, Hélène Beaunieux

\section{- To cite this version:}

Anne-Lise Pitel, Gaël Chételat, Anne-Pascale Le Berre, Béatrice Desgranges, Francis Eustache, et al. Macrostructural abnormalities in Korsakoff syndrome compared with uncomplicated alcoholism.. Neurology, 2012, 78 (17), pp.1330-3. 10.1212/WNL.0b013e318251834e . inserm-00652244

\section{HAL Id: inserm-00652244 https://www.hal.inserm.fr/inserm-00652244}

Submitted on 22 May 2014

HAL is a multi-disciplinary open access archive for the deposit and dissemination of scientific research documents, whether they are published or not. The documents may come from teaching and research institutions in France or abroad, or from public or private research centers.
L'archive ouverte pluridisciplinaire HAL, est destinée au dépôt et à la diffusion de documents scientifiques de niveau recherche, publiés ou non, émanant des établissements d'enseignement et de recherche français ou étrangers, des laboratoires publics ou privés. 


\section{Macrostructural abnormalities in Korsakoff's syndrome compared to} uncomplicated alcoholism

AL Pitel (PhD), G Chételat (PhD), AP Le Berre (PhD), B Desgranges (PhD), F Eustache (PhD), H Beaunieux (PhD)

INSERM, U1077, F-14033 Caen, France

EPHE, UMR-S1077, F-14033 Caen, France

Université de Caen/Basse-Normandie, UMR-S1077, F-14033 Caen, France

CHU de Caen, UMR-S1077, F-14033 Caen, France

Word count for the title: 9

Word count for the abstract: 221

Word count for the paper: 1353

Number of references: 10

Number of tables and figures: 2

Supplemental data:2 figures

The statistical analyses were conducted by AL Pitel and G Chételat

Study funding: supported by Inserm, ANR retour Post-doctorant 2010 and PHRC Inter-régionalKorsakol

Search terms: Alcohol [258], MRI [120], Memory [201] 
Corresponding author: AL Pitel, Inserm-EPHE-Université de Caen/BasseNormandie, Unité U1077, GIP Cyceron, Bd H. Becquerel, BP 5229, 14074 Caen Cedex, France; E-mail: pitel@cyceron.fr

\section{Author contribution}

AL Pitel: study concept and design, statistical analyses and interpretation of data, writing of the manuscript

G Chételat: statistical analyses and interpretation of data, revising the manuscript for content

AP Le Berre: revising the manuscript for content

B Desgranges: study concept and design, revising the manuscript for content F Eustache: study concept and design, revising the manuscript for content H Beaunieux: study concept and design, revising the manuscript for content

\section{Disclosure}

None of the authors disclaim disclosures. 


\section{Abstract}

Objective:Todisentangle, among the structural damage observed in Korsakoff's syndrome (KS), those related to alcoholism, and therefore shared between $\mathrm{AL}$ and KS, from thosespecific to KS.

Methods: Magnetic resonance imaging (MRI) data were collected in11alcoholic patients with KS, 34 non-Korsakoff alcoholic patients $(A L)$ and 25 healthy control subjects (CS). Gray and white matter volumes were compared in the three groups using a voxel-based approach.

Results: Aconjunction analysis indicated a large pattern of shared gray and white matter volume deficits in AL and KS. There were graded effects of volume deficits $(\mathrm{KS}<\mathrm{AL}<\mathrm{CS}$ ) in the medial portion of the thalami, hypothalamus (mammillary bodies), left insula andgenu of the corpus callosum.Abnormalities in the left thalamic radiation wereobservedonly in KS.

Conclusions: Our results indicate considerable similarities in the pattern of gray and white matter damage in $\mathrm{AL}$ and $\mathrm{KS}$. This finding confirms the widespread neurotoxic effect of chronic alcohol consumption. Only a few cerebral regions, including the medial thalami, mammillary bodies and corpus callosum, were more severely damaged in $\mathrm{KS}$ than in $\mathrm{AL}$. The continuum of macrostructural damage from $\mathrm{AL}$ to $\mathrm{KS}$ is therefore restrictedto key brain structures. Longitudinal investigations are required to determine whether $\mathrm{AL}$ with medial thalamic volumes that are comparable to those of KS are at increased risk to develop Korsakoff's syndrome. 


\section{Introduction}

Korsakoff's syndrome (KS) is characterized by a disproportionate impairment of episodic memory compared with other aspects of cognitive function (1).Itresults from the combinationof heavy alcohol consumptionand thiamine deficiency.

Macrostructural abnormalities were initially examined by neuropathological studies, which concluded that specific thalamic nuclei and mammillary bodies play a key role in the pathophysiology of $\mathrm{KS}(2,3)$.In vivo neuroimaging investigations confirmed reduced volume in thalami and mammillary bodies in $\mathrm{KS}(4,5)$ and revealed widespread cerebral damage with the nodes and connections of thefronto-cerebellar and limbic circuits being especially affected(6). These circuits are also classically described damaged in non-Korsakoffalcoholics (AL;(7)). A direct comparison of brain damage in KS and ALwas conductedin several regions of interest and revealed acontinuum of brain damage, from mild in $\mathrm{AL}$ to moderate or severe in $\mathrm{KS}$, notably in the mammillary bodies and thalami(8). However,the comparison between $A L$ and $K S$ has never been conducted throughout the whole brain. The goalof the present study was thusto compare gray and white matter abnormalities in patients with AL and KS using a voxelwise approach to disentangle brain damage related to chronic alcohol consumption, and therefore shared between $\mathrm{AL}$ and $\mathrm{KS}$, from those selectively damaged in KS.

\section{Method}

Sample

Eleven patients with KS ( 5 women, age $=56.00 \pm 10.55)$, 34 patients with AL (6 women, age $=43.47 \pm 8.36)$ and 25 healthy control subjects (CS, 14 women, age $=43.88 \pm 11.24$ ) were enrolled. None of the participants were taking psychotropic 
medication, or presented withhistory of psychiatricor medical problems (head injury, coma, epilepsy, depression, hepatic encephalopathy etc.),which might affect cognitive function. KS patients were older than $\mathrm{AL}$ and controls $(\mathrm{F}=7.37, p=0.001$; Tukey's test: $K S>$ controls, $p=0.003$ and $K S>A L, p=0.001$ ).

KS patients were diagnosed with reference to the DSM IVcriteria for "Persisting Amnestic Disorder". A detailed neuropsychological examination confirmed that all KSpresenteddisproportionately severe episodic memory disorders comparedwith other cognitive functions (see (6)for more details). All KS patients had a history of heavy drinking but it was difficult to gain an accurate picture of their drinking history because of the amnesia.

AL patients were recruited by clinicians on the basis of the DSM-IV criteria for alcohol dependencewhile they were receiving treatment for alcohol dependence as inpatients at Caen University Hospital(see (9)for more details). All patients were at an early stage of abstinence (12.67 \pm 6.94 days of sobriety prior to inclusion). They were interviewed to specify the length of time they had drunk to excess $(16.09 \pm 10.29$ years) and their usual daily alcohol consumption (23.36 \pm 15.01 "standard drinks" per day, i.e. any drink that contains approximately 10 grams of pure alcohol). Controls were social drinkers as defined by the Nationallnstitute on Alcohol Abuse and Alcoholism.

Standard Protocol Approvals, Registrations, and Patient Consents The participants gave their informed consent prior to their inclusion in the study, which was conducted in line with the Declaration of Helsinki and was approved by the local ethical committee for human investigations. 


\section{MRI acquisition and preprocessing}

For each subject, a high-resolution T1-weighted volumeMRI scan was obtained, which consisted of a set of 128 adjacentaxial slices parallel to the AC-PC line, covering the whole brainand with a $1.5-\mathrm{mm}$ slice thickness and a $0.94 \times 0.94 \mathrm{~mm}$ pixel size, through the use ofthe spoiled gradient echo sequence $(T R=10.3 \mathrm{~ms} ; \mathrm{TE}=2.1$ $m s ; F O V=240 \times 180 \mathrm{~mm} 2$; matrix $=256 \times 192)$. All theMRI datasets were acquired on the same scanner (1.5T SignaAdvantage Echospeed; General Electric), and based onthe sameacquisition protocol.

The MRI data were analyzed according tothe VBM5 toolbox implemented in Statistical Parametric Mapping 5 software (SPM5; Wellcome department of cognitive Neurology, Institute of Neurology, London, England). Briefly, the procedure included segmentation, registration (spatial normalization) of original MRI datasetsusing the default MNI template of SPM5 as priors,modulation (to correct only for non-linear warping so that values in the resultant images are expressed as volume corrected for global brain size) andsmooth(10 mm Gaussian kernel).

\section{Statistical analyses}

Preprocessed data were analyzed in SPM5 in twofull factorial designs (for gray and white matter independently) using age and gender as covariates. We first examinedregions with reduced volume in each patient group compared to controls. We then conducted a conjunction analysis of the two previous comparisons $(A L<C S$ andKS $<\mathrm{CS}$ )to reveal brain abnormalities shared betweenthe two patient groups.For these analyses, we used a $p$ value of $p<0.05$ corrected for False Discovery Rate(FDR). We thenassessed whether there was any area of significantly greater brain damage in KS compared to AL (within the regions significantly damaged in KS 
compared to controls). We thus assessed the contrast KS-AL with the contrast KSCS as a mask and using a less stringent $p$ value $p<0.001$ uncorrected for multiple comparisons. Indeed, the differences between these two pathologies are more subtle than the differences between each of these pathologies and the controls, so that a more permissive threshold should be employed to avoid false negative. When necessary, average signal within significant clusters were extracted and post-hoc tests (Tukey's test) were conducted. For all analyses, we used an extended threshold of 200 voxels.

A confirmatory analysiswas also conducted in age-matched subgroups of participants with equal sample sizes (supplementary data e-1).

\section{Results}

Analyses of gray matter volume

As illustrated in Figure $1 \mathrm{~A}$ and $\mathrm{B}$, the analysis revealed widespread gray matter damage in both $\mathrm{AL}$ and $\mathrm{KS}$ patientscompared tocontrols. The conjunction analysis indicated extended areas of shared gray matter volume deficitsbetweenAL and KS patients, notably bilaterally in the orbitofrontal cortex extending to the parietal lobe, and in the cingulate cortex, insula, medial temporal lobes, thalami,hypothalamus, and cerebellum $(p<0.05$ FDR corrected, Figure 1C).Graded effects of volume deficits(KS $<A L<C S ; p<0.01$ in all cases)were found in the medial portion of the thalami, hypothalamus (mammillary bodies) and left insula (Figure 2A). Using a $6 \mathrm{~mm}$ Gaussian kernel yielded similar results.

Figure 1 about here 
Analyses of white matter volume

Figure $1 \mathrm{D}$ and $\mathrm{E}$ illustrates white matter volume deficits in $\mathrm{AL}$ and $\mathrm{KS}$ patients compared tocontrols. The conjunction analysis revealedsharedwhite matter volume deficits in $\mathrm{AL}$ and $\mathrm{KS}$ patients in the corpus callosum and cerebellar peduncles and bilaterally in the fornix and cingulum (Figure 1F). There were graded effects of brain damage in the corpus callosum ( $\mathrm{KS}<\mathrm{AL}<\mathrm{CS}$; $\mathrm{p}<0.01$ in all cases) but the left thalamic radiation was exclusively affected in the KS (Figure 2B).

Figure 2 about here.

\section{Discussion}

The present voxel-based comparison of gray and white matter indicatedstriking similarities in the regional distribution and severityof brain damage in $\mathrm{AL}$ and $\mathrm{KS}$. This finding confirms the widespread direct or indirect (dietary deficiencies) neurotoxic effects of chronic alcohol consumption on the structure of the brain. The frontocerebellar circuit was affected to the same extent in AL and KS, in agreement with neuropsychological data collected in other groups of $A L$ and KS, whichindicatedsimilar pattern of working memory deficits and executive dysfunction in each of the two groups(10). Even though we found volume deficits in Papez's circuit in both patient groups, this circuit was more severely damagedin $\mathrm{KS}$ than in $\mathrm{AL}$ in agreement with a previous neuroimaging investigation (8). Moreover, the left thalamic radiation was the only brain region to be exclusively altered in $\mathrm{KS}$ (with the statistical threshold used here). These findings fit with the fact that episodic memory is impaired both in $\mathrm{AL}$ and $\mathrm{KS}$ but that the main neuropsychological feature that distinguishes $A L$ and $K S$ is the severity of amnesia (10). 
Only a few cerebral regions, including the medial thalami, mammillary bodies and corpus callosum, were more severely damaged in $\mathrm{KS}$ than in $\mathrm{AL}$, suggesting that the continuum of macrostructural damage from $A L$ to $K S$ is selective to key brain structures. Statistical cluster analyses (Supplementary data e2) indicated that alcoholics with medial thalamic volume inferior to 2 standard deviations from controls were included within the same cluster as KS patients. Longitudinal investigations are required to determine whether those $\mathrm{AL}$ are at risk to develop Korsakoff's syndrome. 


\section{References}

(1) Kopelman MD. The Korsakoff syndrome. Br J Psychiatry 1995;166:154-173.

(2) Victor M, Adams RD, Collins GH. The Wernicke-Korsakoff syndrome. A clinical and pathological study of 245 patients, 82 with post-mortem examinations. Contemp Neurol Ser 1971;7:1-206.

(3) Sheedy D, Lara A, Garrick T, Harper C. Size of mamillary bodies in health and disease: useful measurements in neuroradiological diagnosis of Wernicke's encephalopathy. Alcohol Clin Exp Res 1999;23:1624-1628.

(4) Shimamura AP, Jernigan TL, Squire LR. Korsakoff's syndrome: radiological (CT) findings and neuropsychological correlates. J Neurosci 1988;8:44004410.

(5) Sullivan EV, Lane B, Deshmukh A, et al. In vivo mammillary body volume deficits in amnesic and nonamnesic alcoholics. Alcohol Clin Exp Res 1999;23:1629-1636.

(6) Pitel AL, Aupée AM, Chételat G, et al. Morphological and glucose metabolism abnormalities in alcoholic Korsakoff's syndrome: group comparisons and individual analyses. PLoS One 2009;4:e7748.

(7) Chanraud S, Martelli C, Delain F, et al. Brain morphometry and cognitive performance in detoxified alcohol-dependents with preserved psychosocial functioning. Neuropsychopharmacology 2007;32:429-438.

(8) Sullivan EV, Pfefferbaum A. Neuroimaging of the Wernicke-Korsakoff syndrome. Alcohol Alcohol 2009;44:155-165. 
(9) Le Berre AP, Pinon K, Vabret F, et al. Study of metamemory in patients with chronic alcoholism using a feeling-of-knowing episodic memory task. Alcohol Clin Exp Res 2010;34:1888-1898.

(10) Pitel AL, Beaunieux H, Witkowski T, et al. Episodic and working memory deficits in alcoholic Korsakoff patients: the continuity theory revisited. Alcohol Clin Exp Res 2008;32:1229-1241. 
Acknowledgement: AM Aupéefor data collection in the KS group,Vincent de La

Sayette, François Vabret and FaustoViader for screeningpatients with alcoholism and Korsakoff's syndromeandEric MacKenzie for improving the English style 


\section{Figure 1: Gray and white matter abnormalities in AL, KS and shared between}

\section{$\mathrm{AL}$ and $\mathrm{KS}$}

KS:alcoholics with Korsakoff's syndrome; AL:non-Korsakoff alcoholics; CS: control subjects
$A: A L<C S ; B: K S<C S ; C: A L<C S$ and $K S<C S$ (gray matter)
$D: A L<C S ; E: K S<C S ; F: A L<C S$ and $K S<C S$ (white matter)

We used a $p$ value cut-off of $p<0.05$ corrected for False Discovery Rate (FDR, larger images) but also displayed the results using a restrictive $p<0.05$ corrected for FamilyWise Error (FWE, smaller images)to highlight the most significant regions.

Cluster size $>200$ voxels

Figure 2: Gray (A) and white (B) matter regions more severely damaged in

\section{KSthan in AL}

KS:alcoholics with Korsakoff's syndrome; AL:non-Korsakoff alcoholics; CS: control subjects

For the gray and white matter clusters that most significantly differentiate AL and KS, a $3 \mathrm{D}$ representation of the brain region and results of the post-hoc comparisons are also provided. The average signal within the cluster was used for this analysis.

$\mathrm{P}<0.001$ uncorrected; Cluster size $>200$ voxels

*: significant difference compared to controls

†: significant difference compared to alcoholics 